PROCEEDINGS OF THE

AMERICAN MATHEMATICAL SOCIETY

Volume 139, Number 5, May 2011, Pages 1521-1532

S 0002-9939(2010)10572-5

Article electronically published on September 15, 2010

\title{
WEIGHTED SHORT-INTERVAL CHARACTER SUMS
}

\author{
SHIGERU KANEMITSU, HAILONG LI, AND NIANLIANG WANG
}

(Communicated by Wen-Ching Winnie Li)

Dedicated to Professor Masaaki Yoshida on his sixtieth birthday

with great respect and friendship

\begin{abstract}
In this paper we shall establish the counterpart of Szmidt, Urbanowicz and Zagier's formula in the sense of the Hecker correspondence. The motivation is the derivation of the values of the Riemann zeta-function at positive even integral arguments from the partial fraction expansion for the hyperbolic cotangent function (or the cotangent function). Since the last is equivalent to the functional equation, we may view their elegant formula as one for the Lambert series, and comparing the Laurent coefficients, we may give a functional equational approach to the short-interval character sums with polynomial weight.

In view of the importance of these short-interval character sums, we assemble some handy formulations for them that are derived from Szmidt, Urbanowicz and Zagier's formula and Yamamoto's method, which also gives the conjugate sums. We shall also state the formula for the values of the Dirichlet $L$-function with imprimitive characters.
\end{abstract}

\section{INTRODUCTION}

Our aim in this paper is twofold.

First we shall give the counterpart (our Theorem 3 in $\S 4$ ) of Szmidt, Urbanowicz and Zagier's important formula for the polynomial weight short-interval character sum ([35, (6)]) stated as Formula (2.11) in $\S 2$ of the present paper, in the sense of the Hecke correspondence. The Hecke (or Riemann, Hecke and Bochner) correspondence is the correspondence of the transformation formula for the modular form (Lambert series) and the functional equation for the zeta (or $L$ )-function. This correspondence can be described as one for the Mellin and the inverse Mellin transform (cf. e.g. [8], 23, 26, etc.). For some more information on this, we refer to $\S 4$. Since we may deduce (2.10) and thence (2.11) from the functional equation, we may claim that the short-interval character sums can be treated by the functional

Received by the editors October 28, 2009 and, in revised form, February 11, 2010 and May 4, 2010 .

2010 Mathematics Subject Classification. Primary 11L03, 11L26; Secondary 11B68, 11T24, $11 \mathrm{~S} 40$.

Key words and phrases. Dirichlet characters, Lambert series, Bernoulli numbers, short-interval character sums.

The authors were supported in part by JSPS grant No. 21540029 and by the NSF of Shaanxi Province (No. 2010JM1009). 
equation. We have come upon this standpoint by viewing Szmidt, Urbanowicz and Zagier's formula as a form of a Lambert series.

At the time when we wrote the first version of the present paper, we thought we would need the functional equation for the Hurwitz-Lerch $L$-function introduced by Morita 31] (for this, cf. the proof of Theorem 33), but it has turned out that we need that of the Dirichlet $L$-function and the complete character sum (for applications to short-interval sums). Thus we are led to give formulas for the special values of the relevant $\ell$-function (cf. (2.5)) at positive integer arguments, which is essential in the case of imprimitive characters, whence those for the Dirichlet $L$-functions with imprimitive characters. This part is also based on Riemann, Hecke and Bochner's correspondence.

Secondly, we will state some concrete formulas for the short-interval character sums with imprimitive characters for convenience of reference. This is because, to treat such sums, most of the authors refer to [7] or [18] but not [35], [38, or more recently [34; cf. [37] for examples of such redundancies. Most of the literature contains formulas for primitive characters but one sometimes needs formulas for imprimitive characters as in the case of the analytic-number-theoretic application of discrete mean squares (cf. e.g. [22]) in which one needs a formula valid for all characters in order to use the orthogonality. In 38] and 34] the authors' intension was to apply the results to the quadratic fields and therefore all the characters they need are primitive Kronecker characters. Since Szmidt, Urbanowicz and Zagier's formula is valid for all characters, we are led to trying to generalize Yamamoto's method to this case. We note that Yamamoto's method allows us to treat shortinterval character sums with Clausen function weight (log sin integrals, cf. [30]) as well, which enables us to obtain class number relations for real quadratic fields such as those in [13, 29, 38. Thus it is rather important and intriguing to generalize the method of Yamamoto to the case of imprimitive characters, which we expect to accomplish duly elsewhere.

\section{Szmidt, Urbanowicz And ZagieR's FORMUla}

In this section we present Szmidt, Urbanowicz and Zagier's elegant formula 35. (Formula (2.10) below) and show that it is in spirit exactly the same as the classical method for obtaining the values of the Riemann zeta-function at even positive integral arguments. Indeed, it leads to the most well-known formula of Euler.

Euler's formula,

$$
\frac{B_{2 m}}{(2 m) !}=(-1)^{m-1} \frac{2 \zeta(2 m)}{(2 \pi)^{2 m}}, \quad m \geq 1,
$$

follows from the partial fraction expansion for the hyperbolic cotangent function

$$
\frac{1}{2} \operatorname{coth} \pi x=\frac{1}{e^{2 \pi x}-1}+\frac{1}{2}=\frac{1}{2 \pi x}+\frac{x}{\pi} \sum_{n=1}^{\infty} \frac{1}{n^{2}+x^{2}}, \quad \operatorname{Re} x \geq 0 .
$$

The proof can be found in [10, pp. 39-43], [33, p. 91], [24, Exercise 5.4], but we indicate it here. Rewriting (2.1) in the form

$$
\frac{z}{e^{z}-1}=1-\frac{1}{2} z+2 z^{2} \sum_{n=1}^{\infty} \frac{1}{z^{2}+(2 \pi n)^{2}}
$$


and expanding the infinite series into the Taylor series, we deduce (cf. [35, (6)]) that

$$
\frac{z}{e^{z}-1}=1-\frac{1}{2} z+\sum_{m=1}^{\infty} \frac{2(-1)^{m-1}}{(2 \pi)^{2 m}} \zeta(2 m) z^{2 m}, \quad|z|<2 \pi,
$$

i.e. the zeta-value coefficients. On the other hand, by definition, the left-hand side has the well-known expansion (which is a generating function of the Bernoulli numbers)

$$
\frac{z}{e^{z}-1}=1-\frac{1}{2} z+\sum_{m=1}^{\infty} \frac{B_{2 m}}{(2 m) !} z^{2 m}, \quad|z|<2 \pi .
$$

Comparing the coefficients, we deduce Euler's formula.

Here we note that the partial fraction expansion for the hyperbolic cotangent function is equivalent to the functional equation for the Riemann zeta-function, whose asymmetric form reads

$$
\zeta(1-s)=2^{1-s} \pi^{-s} \Gamma(s) \cos \left(\frac{\pi s}{2}\right) \zeta(s),
$$

which is a consequence of the functional equation for the Dirichlet $L$-function for the trivial primitive Dirichlet character taking the value 1 for all integers.

Now Joris [19] (cf. also 32, 5]) states the functional equation

$$
L(1-s, \chi)=M^{s-1}(2 \pi)^{-s} \Gamma(s)\left\{e^{-\frac{\pi i}{2} s}+\chi(-1) e^{\frac{\pi i}{2} s}\right\} \ell(s, \chi),
$$

where

$$
\ell(s, \chi)=\sum_{a \bmod M} \chi(a) l_{s}\left(\frac{a}{M}\right)
$$

and where $l_{s}(x)$ indicates the polylogarithm function defined by

$$
l_{s}(x)=\sum_{n=1}^{\infty} \frac{e^{2 \pi i n x}}{n^{s}}, \sigma>1, a \in \mathbb{R}(\text { or } s=1,0<x<1) .
$$

In view of [19, 32, pp. 211-215] and [27, we see that the essential ingredient is this $\ell$-function in the study of $L$-functions with imprimitive characters. For further use of this $\ell$-function, see the last half of $\S 4$.

Throughout the paper whenever we refer to a character, we mean a Dirichlet character that is not necessarily primitive.

We assemble here some data on the generalized Bernoulli numbers and polynomials introduced by Szmidt, Urbanowicz and Zagier. First, let $B_{n, \chi}\left(\right.$ and $B_{n, \chi}(x)$ ) denote the well-known ordinary generalized Bernoulli numbers (and polynomials) whose properties are contained in them. All these may conveniently be introduced by the generating function similar to (2.3) (cf. also [17, 28], etc.):

$$
B_{n, \chi}^{[r]}=\prod_{p \mid r, p \text { prime }}\left(1-\chi(p) p^{n-1}\right) \cdot B_{n, \chi} \quad(0 \neq r \in \mathbb{Z}) ;
$$

i.e. $B_{n, \chi}^{[r]}=B_{n, \chi^{\prime}}$ for the character $\chi^{\prime}$ modulo $M|r|$ induced by $\chi$ (whence $B_{n, \chi}^{[1]}=$ $\left.B_{n, \chi}\right)$. Conversely, the special case, where $\chi$ is induced by the primitive character $\psi$ modulo $f$ is the most essential: $B_{n, \psi}^{[M / f]}=B_{n, \chi}$. Second,

$$
B_{n, \chi}^{[r]}(x)=\sum_{k=0}^{n}\left(\begin{array}{l}
n \\
k
\end{array}\right) B_{k, \chi}^{[r]} x^{n-k}
$$


so that $B_{n, \chi}^{[r]}=B_{n, \chi}^{[r]}(0)$. The properties $B_{n, \chi}^{[r]}(-x)=(-1)^{n} \chi(-1) B_{n, \chi}^{[r]}(x)$ unless $M=n=r=1$ imply that $B_{n, \chi}=0$ if $n>1, \chi(-1)=(-1)^{n-1}$ and that $B_{0, \chi}=0$ for non-principal $\chi$. If $\chi$ is the principal character, then $B_{0, \chi}=\frac{\varphi(M)}{M}$.

The following formula gives the value of the $L$-function at negative integers:

$$
L(1-n, \chi)=-\frac{B_{n, \chi}}{n}(n \geq 1)
$$

which in turn leads to the evaluation at positive integer arguments of the $L$-function in Theorem 4 below.

For a Dirichlet character $\chi$ modulo $M$, let $L_{\chi}(t)$ define the Lambert series associated to $\chi$ :

$$
L_{\chi}(t)=\sum_{n=1}^{\infty} \chi(n) e^{-n t}, \quad \operatorname{Re} t>0
$$

which corresponds to the hyperbolic cotangent function above. For references on Lambert series with or without characters, we refer to $\S 4$.

Let $N$ be a multiple of $M$, say $N=u M$, and let $r$ be a positive integer prime to $N$. The essential case is $u<r$, which we so assume. Then Szmidt, Urbanowicz and Zagier [35, p. 275] deduced the elegant formula

$$
\sum_{0<n<\frac{N}{r}} \chi(n) e^{-r n t}=L_{\chi}(r t)-\frac{\bar{\chi}(r)}{\varphi(r)} e^{-N t} \sum_{\psi \bmod r} \bar{\psi}(-N) L_{\chi \psi}(t)
$$

with $\varphi$ being the Euler $\varphi$-function. This corresponds to the transformation formula for the Lambert series (2.2).

With the above $M, N, r, u$ we define the short-interval character sum with polynomial weight

$$
S_{r, N}^{k}(\chi)=S_{r, u M}^{k}(\chi)=\frac{1}{M^{k}} \sum_{1 \leq a \leq \frac{N}{r}}^{\prime} \chi(a) a^{k}
$$

In the notation of Yamamoto [38, p. 280], this is $S_{\frac{u}{r}}=S_{\underline{N / M}}$.

Comparing the coefficients of both sides of (2.10) and using the Laurent expansion ([35, (5)]) of (2.9), Szmidt, Urbanowicz and Zagier [35, (6), p. 276] deduced that

$$
(k+1)(r M)^{k} S_{r, N}^{k}(\chi)=-B_{k+1, \chi} r^{k}+\frac{\bar{\chi}(r)}{\varphi(r)} \sum_{\psi} \bar{\psi}(-N) B_{k+1, \chi \psi}(N) .
$$

Thus we are naturally led to the consideration of the relation for the $L$-function, corresponding to (2.11) in view of the presence of the functional equation (2.4) (to be given in $\S 4$ ). We note that the Laurent expansion [35, (5)] is a limiting relation of the Lambert series as the variable tends to the real axis through the upper half-plane.

For convenience of reference, we state two similar formulations of Szmidt, Urbanowicz and Zagier's formula (2.11) which are equivalent under the following relation. 
Assume that $\chi$ modulo $M$ is induced by the primitive character $\psi$ to the conductor $f>1$. Then we have

$$
B_{n, \chi}= \begin{cases}0 & \text { if } \chi(-1)=(-1)^{n-1}, \\ \frac{(-1)^{n-1} n ! f^{n}}{(2 \pi i)^{n} \tau(\psi)} \prod_{p \mid M}\left(1-\psi(p) p^{n-1}\right) L(n, \psi) & \text { if } \chi(-1)=(-1)^{n},\end{cases}
$$

where $\tau(\chi)=\sum_{a=1}^{M} \chi(a) e^{\frac{2 \pi i a}{M}}$ is the normalized Gauss sum (cf. Lemma 1 below).

(i) Assume that $u<r$ are positive integers and $\chi$ is a Dirichlet character modulo $M$ such that $(u M, r)=1$. Then

$$
\begin{aligned}
S_{r, u M}^{k}(\chi)= & -\frac{1}{M^{k}} \frac{B_{k+1, \chi}}{k+1}+\frac{\bar{\chi}(r)}{(r M)^{k} \varphi(r)} \sum_{\psi} \bar{\psi}(-u M) \\
& \times \sum_{a=1}^{k+1} \frac{1}{k+1}\left(\begin{array}{c}
k+1 \\
a
\end{array}\right)(u M)^{k+1-a} B_{a, \chi \psi},
\end{aligned}
$$

where the sum is over all Dirichlet characters $\psi$ modulo $r$.

(ii) Assume in addition that $\chi$ is induced by the primitive character $\chi_{1}$ to the conductor $f$. Then we have

$$
\begin{aligned}
S_{r, u M}^{k}(\chi)= & -\frac{(-1)^{k+1}+\chi(-1)}{2(k+1) M^{k}} \frac{(-1)^{k}(k+1) ! f^{k+1}}{(2 \pi i)^{k+1} \tau\left(\chi_{1}\right)} \prod_{p \mid M}\left(1-\chi_{1}(p) p^{k}\right) L\left(k+1, \chi_{1}\right) \\
& +\frac{\bar{\chi}(r)}{(M N)^{k} \varphi(N)} \sum_{\psi} \bar{\psi}(-u M) \sum_{a=1}^{k+1} \frac{1}{k+1}\left(\begin{array}{c}
k+1 \\
a
\end{array}\right)(u M)^{k+1-a} \\
& \times \frac{(-1)^{a}+\chi \psi(-1)}{2} \frac{(-1)^{a-1} a ! f^{\prime a}}{(2 \pi i)^{a} \tau\left((\chi \psi)_{1}\right)} \prod_{p \mid r M}\left(1-(\chi \psi)_{1}(p) p^{a-1}\right) L\left(a,(\chi \psi)_{1}\right)
\end{aligned}
$$

where the sum is over all Dirichlet characters $\psi$ modulo $r$ and each $\chi \psi$ is induced from $(\chi \psi)_{1}\left(\bmod f^{\prime}\right)$.

\section{YAMAMOTO's METHOD}

It seems that Yamamoto's pretty results 38 are less well-known. In this section we present them with details.

One writes

$$
S_{r, u M}^{k}(\chi)=\sum_{a=1}^{M} \chi(a) f\left(\frac{a}{M}\right)
$$

where

$$
f(x)=\left\{\begin{array}{cc}
x^{k} & \left(0<x \leq \frac{u}{r}\right), \\
0 & \left(\frac{u}{r}<x \leq 1\right),
\end{array}\right.
$$

and uses the Fourier series for $f$. His results are limited to the case of primitive characters, but could hopefully be generalized by using the following general evaluation of the Gauss sum $G_{n}(\chi)$ in Joris [19] (cf. also Hasse [15, pp. 444-450]). For primitivity of $\chi$ and the separability of the Gauss sum, cf. [1], [2], 3], 6]. We now state an equivalent form of Yamamoto's results in terms of Joris's evaluation of the 
Gauss sum. We write the primitive character inducing $\chi$ to the modulus $M$ by $\psi$ with modulus $f$ and introduce the notation

$$
\tilde{q}=\prod_{\substack{p \mid M \\ p \backslash f}} p, \quad R=\frac{M}{f \tilde{q}} .
$$

Lemma 1 ([19, p. 14]).

$$
G_{n}(\chi)= \begin{cases}0 & \text { if } R \nmid n, \\ \mu(\tilde{q}) \varphi(\tilde{q}) \tau(\psi) R g\left(\frac{n}{R}\right) & \text { if } R \mid n,\end{cases}
$$

where

$$
g(n)=\mu((n, \tilde{q})) \varphi((n, \tilde{q})) \bar{\psi}(n) .
$$

Theorem 1. The notation being the same as above, we have

$$
\begin{aligned}
S_{r, u M}^{k}(\chi) & =R \mu(\tilde{q}) \psi(\tilde{q}) \\
& \times \sum_{m=1}^{k+1} \frac{m !\left(\frac{u}{r}\right)^{k-m+1} \tau(\psi)}{(2 \pi i)^{m}(k-m+1) !} \sum_{\substack{n=1 \\
R \mid n}}^{\infty} \mu((n / R, \tilde{q})) \varphi((n / R, \tilde{q})) \bar{\psi}(n / R) \frac{b_{m}(n)}{n^{m}},
\end{aligned}
$$

where $b_{m}(n)$ are defined by

$$
\begin{aligned}
& b_{m}(n)=(-1)^{m+1} \chi(-1) \eta^{n u}-\eta^{-n u} \quad(1 \leq m \leq k), \\
& b_{k+1}(n)=(-1)^{k+1} \chi(-1)\left(1-\eta^{n u}\right)+1-\eta^{-n u}, \eta=e^{\frac{2 \pi i}{r}} .
\end{aligned}
$$

Indeed, Theorem 1 is in its intermediate stage, [38, (5.3)], and the final form is [38, Theorem 5.1], which expresses $S_{r, u M}^{k}(\chi)$ in terms of the $L\left(m,(\chi \psi)_{1}\right)$ 's. It is easy to prove that (2.12) and (2.13) and [38, Theorem 5.1] are equivalent, provided that the coefficients $b_{r, \psi}([38$, p. 281]) are given explicitly. It has turned out that it is far simpler to work out the series of the form $\sum_{n=1}^{\infty} \frac{b_{m}(n)}{n^{m}}$ as has been done in [38] and 37. Thus we leave Theorem 1 at that and expect on another occasion to work out the explicit coefficients and prove the equivalence of these theorems.

Yamamoto also defined the conjugate character sum $T_{r, u M}^{k}(\chi)$ :

$$
T_{r, N}^{k}(\chi)=T_{r, u M}^{k}(\chi)=\sum_{a=0}^{M-1} \chi(a) \tilde{f}\left(\frac{a}{M}\right),
$$

for a character $\chi$ modulo $M>1$, where $\tilde{f}$ is the conjugate function of $f$ defined by (cf. [38, p. 285])

$$
\tilde{f}(x)=P V \int_{0}^{1} f(x-y) \cot \pi y \mathrm{~d} y,
$$

which has the Fourier series

$$
\tilde{f}(x) \sim-i \sum_{n=-\infty}^{\infty} \operatorname{sgn}(\mathrm{n}) \tilde{f}_{n} e^{2 \pi i n x}
$$

converging to $\tilde{f}(x)$ for every $x \neq 0, \frac{u}{r}$.

Yamamoto's method gives a general evaluation for $T_{r, u M}^{k}(\chi)$. For comparison with Theorem 1 we state the theorem using the evaluation of the general Gauss sum by Hasse [15]. We write for $n \in \mathbb{N}, n_{0}=\frac{n}{(M, n)}, M_{0}=\frac{M}{(M, n)}$. 


\section{Lemma 2.}

$$
G_{n}(\chi)= \begin{cases}0 & \text { if } f \nmid M_{0}, \\ \frac{\varphi(M)}{\varphi\left(M_{0}\right)} \mu\left(\frac{M_{0}}{f}\right) \psi\left(\frac{M_{0}}{f}\right) \bar{\psi}\left(n_{0}\right) \tau(\psi) & \text { if } f \mid M_{0} .\end{cases}
$$

Theorem 2. Let $\chi, f, \psi, M, M_{0}, n_{0}, \mu, \varphi$ be the same as above and assume $M \nmid N$. Then

$$
T_{r, N}^{k}(\chi)=-i \sum_{m=1}^{k+1} \frac{\left(\frac{u}{N}\right)^{k-m+1} k ! \varphi(M) \tau(\psi)}{(2 \pi i)^{k+1}(k-m+1) !} \sum_{n=1, f \mid M_{0}}^{\infty} \mu\left(\frac{M_{0}}{f}\right) \psi\left(\frac{M_{0}}{f}\right) \frac{\bar{\psi}\left(n_{0}\right)}{\varphi\left(M_{0}\right)} \frac{\tilde{b}_{m}(n)}{n^{m}}
$$

where $b_{m}$ are given by (3.2).

Proof. We note that the sum $T_{r, N}^{k}(\chi)$ is taken over $(a, M)=1$, and we have the Fourier expansion:

$$
\begin{aligned}
T_{r, N}^{k} & =-i \sum_{a=0}^{M-1} \chi(a) \sum_{n=-\infty}^{\infty} \operatorname{sgn}(n) \hat{f}_{n} e^{\frac{2 \pi i n a}{M}} \\
& =-i \sum_{n=1}^{\infty}\left(\hat{f}_{n}-\chi(-1) \hat{f}_{-n}\right) G_{n}(\chi) \\
& =-i \sum_{m=1}^{k+1} \frac{\left(\frac{u}{N}\right)^{k-m+1} k !}{(2 \pi i)^{k+1}(k-m+1) !} \sum_{n=1}^{\infty} \frac{G_{n}(\chi) \tilde{b}_{m}(n)}{n^{m}},
\end{aligned}
$$

where $\tilde{b}_{m}(n)$ is defined in a similar way to (3.2), and substituting (3.3) we conclude (3.4), completing the proof.

\section{The MAIN RESUlts OF THE PAPER}

In this section we prove Theorem 3 below, our main result, forming Riemann, Hecke and Bochner's correspondence (cf. Knopp 26]) with Szmidt, Urbanowicz and Zagier's formula (2.10), by applying the Mellin transform technique. This aspect is already stated in [35, p. 275, 1. 8] to deduce their formula (5), in the spirit of Riemann, splitting up the range of the integral. Riemann already used this technique in his most celebrated paper on the distribution of primes, which was taken up by Hecke to establish the Hecke correspondence between modular forms and zeta-functions (in the case of a single gamma factor). Weil also made some contributions to this area (Vol. 3 of his Collected Papers, in which he refers to Bochner's work), and eventually Bochner [9] treated the case of multiple gamma factors, creating the notion of modular relations. Cf. $\S 5$ for relevant references and some comments.

This depends on the (Hecke) gamma transform

$$
\Gamma(s) n^{-s}=\int_{0}^{\infty} e^{-n t} t^{s} \frac{\mathrm{d} t}{t},
$$

which is valid for $\sigma>0$. Using (4.1), we have for $\sigma>1$,

$$
\Gamma(s) L(s, \chi)=\int_{0}^{\infty} L_{\chi}(s) t^{s} \frac{\mathrm{d} t}{t} .
$$


Theorem 3. Let $\chi$ be a Dirichlet character $\bmod M$, not necessarily primitive. Let $N$ be a multiple of $M$ and let $r>\frac{N}{M}$ be a positive integer prime to $N$. Then we have the modular relation

$$
\sum_{0<n<\frac{N}{r}} \frac{\chi(n)}{n^{s}}=L(s, \chi)-\frac{\bar{\chi}(r)}{\varphi(r)} r^{s} \sum_{\psi} \bar{\psi}(-N)\left(L(s, \chi \psi)-M_{s}(\chi \psi)\right)
$$

valid for all values of $s$, where $M_{s}(\chi)=\sum_{n=1}^{N-1} \frac{\chi(n)}{n^{s}}$ is the weighted complete character sum.

Proof. Taking the Mellin transform of both sides of (2.10) and using (4.2), we obtain for $\sigma>1$,

$$
\Gamma(s) \sum_{0<n<\frac{N}{r}} \frac{\chi(n)}{(r n)^{s}}=\Gamma(s) r^{-s} L(s, \chi)-\Gamma(s) \frac{\bar{\chi}(r)}{\varphi(r)} \sum_{\psi} \bar{\psi}(-N) \Phi(\chi \psi, N, s),
$$

where

$$
\Phi(\chi, a, s)=\sum_{n=0}^{\infty} \frac{\chi(n)}{(n+a)^{s}}
$$

is the Hurwitz-Lerch $L$-function (cf. e.g. [31]), which is analytically continued over the whole plane. Hence the formula

$$
\sum_{0<n<\frac{N}{r}} \frac{\chi(n)}{(r n)^{s}}=r^{-s} L(s, \chi)-\frac{\bar{\chi}(r)}{\varphi(r)} \sum_{\psi} \bar{\psi}(-N) \Phi(\chi \psi, N, s)
$$

is valid for all $s$.

Or more simply, using the expression

$$
\Phi(\chi \psi, N, s)=L(s, \chi \psi)-M_{s}(\chi \psi)
$$

and appealing to the analyticity of the Dirichlet $L$-function furnished by the functional equation (2.4), we deduce (4.3) for all $s \in \mathbb{C}$.

In view of [6, Example 2] with slight modifications (or [35, p. 276]), $M_{s}(\chi)$ may be considered as known for $s=m \in \mathbb{N}$ :

$$
M_{m}(\chi)=\frac{1}{m}\left(B_{m+1, \chi}(M)-B_{m+1, \chi}\right)=\frac{1}{m+1} \sum_{k=1}^{m}\left(\begin{array}{c}
m \\
k
\end{array}\right) B_{m+1-k, \chi} M^{k} .
$$

In view of (4.4), Theorem 3 entails the values at non-positive integers, on which we dwell presently.

Theorem 4. Let $\psi$ denote the primitive character $\psi$ inducing $\chi$ to the modulus M. Then

(i) Under the equality

$$
\prod_{p \mid \tilde{q}}\left(1-\psi(p) p^{-\alpha}\right)=\tilde{q}^{-\alpha} \mu(\tilde{q}) \psi(\tilde{q}) \prod_{p \mid \tilde{q}}\left(1-\bar{\psi}(p) p^{\alpha}\right),
$$

with $\mu$ designating the Möbius function, the following two equations are equivalent:

$$
\ell(s, \chi)=R^{1-s} \tau(\psi) \mu(\tilde{q}) \psi(\tilde{q}) L(s, \bar{\psi}) \prod_{p \mid \tilde{q}}\left(1-\bar{\psi}(p) p^{1-s}\right),
$$

in the notation of (3.1) and

$$
L(s, \bar{\chi})=g(s, \chi) \ell(s, \chi),
$$


where

$$
g(s, \chi)=\frac{1}{\tau(\psi)}\left(\frac{M}{f}\right)^{s-1} \prod_{\substack{p \mid M \\ p \backslash f}} \frac{1-\bar{\psi}(p) p^{1-s}}{1-\psi(p) p^{s-1}} .
$$

(ii) Suppose $\chi$ is a Dirichlet character mod $M$ induced by the primitive character $\psi \bmod f$ and that $\chi(-1)=(-1)^{n}$, where $n$ is a positive integer. Then

$$
L(n, \chi)=-\psi(-1) \frac{B_{n, \bar{\chi}}}{2 n !} \frac{\tau(\psi)(2 \pi i)^{n}}{f^{n}} \prod_{\substack{p \mid M \\ p \backslash f}} \frac{1-\bar{\psi}(p) p^{1-n}}{1-\psi(p) p^{n-1}} .
$$

Proof. Once the linking equality (4.5) is found, (i) is easy to prove. The proof of (ii) is a combination of Joris [19] and Neukirch [32] and depends on the functional equation (2.4) and the special values (2.8), (4.7) and (4.8).

Indeed, using (2.8), (2.4) and (4.7),

$$
-\frac{B_{n, \chi}}{n}=L(1-n, \chi)=M^{n-1}(2 \pi)^{-n}(n-1) ! 2(-i)^{n} g(n, \chi)^{-1} L(n, \chi) .
$$

Hence it follows that

$$
L(n, \chi)=-\frac{B_{n, \chi}}{2 n !} \frac{1}{M^{n-1}} \frac{(2 \pi i)^{n}}{\tau(\psi)}\left(\frac{M}{f}\right)^{n} \prod_{\substack{p \mid M \\ p \backslash f}} \frac{1-\bar{\psi}(p) p^{1-n}}{1-\psi(p) p^{n-1}},
$$

whence, using

$$
\tau(\psi) \tau(\bar{\psi})=\psi(-1) f
$$

we complete the proof.

Remark 1. (i) (4.6) is [19, (7)] and (4.7) is [32, Proposition 4.2]. In [32 $\tau(\psi)$ is written as $\tau(\chi)$. Using (4.5), we get a slightly novel-looking form of (4.9) which however remains within this circle. (ii) It is interesting to notice that several relevant papers, 7], 18], 19] and [38, appeared around the same year (1976) and 7] has been most well-known and most frequently cited.

\section{Conclusion}

In this section we first give the most relevant and informative references on subjects which we referred to in early sections of this paper. Then we state some possible directions of research on weighted short-interval character sums and their applications.

First, for the interpretation of the partial fraction expansion of the cotangent function as an equivalent to the functional equation, we refer to 23. Berndt 4 and Ishii and Oda [16 contain rich references on the transformation formula for the Lambert series, which are mutually independent, with [16] focusing on the special values of a class of zeta-functions.

Second, regarding Riemann, Hecke and Bochner's correspondence, we refer to [23] and the recent books [8] and [12] in addition to [26].

Third, for (generalized) Bernoulli numbers and polynomials we refer to [5], [11, 14] and 36. 
Regarding a possible generalization of Yamamoto's results to the case of imprimitive characters and the possible deduction of Szmidt, Urbanowicz and Zagier's formula from them, we shall wait for another occasion. We have made a slight generalization to the case of imprimitive characters with prime power modulus in [37. to deduce some congruences for Euler numbers. We could pursue this direction with reference to our recent paper [25].

We expect that we could deduce Yamamoto's results for weighted character sums with (primitive) characters of both types from the functional equation in view of the recent results [21, [12]. In these papers we proved that the Fourier expansions of the relevant weight - the Bernoulli polynomial and the Clausen function ( $\log \sin$ integral) - follow from the functional equation, whence Theorems 11 and 2 of Yamamoto.

Regarding the short-interval character sums, there are many applications, including the class number problem. They may be used to obtain congruences for generalized Bernoulli numbers, including Euler numbers modulo a power of a prime (cf. e.g. 25]). In one of our forthcoming papers, we reveal that in P. Chowla's arithmetical formulation of the class number formula for the real quadratic field $\mathbb{Q}(\sqrt{p})[13$, there is no relation between the class numbers of two subfields of the biquadratic field $\mathbb{Q}(i, \sqrt{p})$.

\section{ACKNOWLEDGEMENT}

The authors would like to express their hearty thanks to the referee for a penetrating and comprehensive scrutinization and for excellent suggestions for reorganizing an earlier version of the paper. It was a pleasant duty to improve the paper according to the referee's suggestions.

\section{REFERENCES}

[1] T. M. Apostol, Euler's $\varphi$-function and separable Gauss sums, Proc. Amer. Math. Soc. 24 (1970), 482-485. MR0257006 (41:1661)

[2] T. M. Apostol, Dirichlet L-functions and Dirichlet characters, Proc. Amer. Math. Soc. 31 (1972), 384-386. MR0285499 (44:2717)

[3] T. M. Apostol, Introduction to analytic number theory, Springer-Verlag, Berlin, 1976. MR0434929 (55:7892)

[4] B. C. Berndt, Modular transformations and generalization of several formulas of Ramanujan, Rocky Mount. J. Math. 7 (1977), 147-189. MR0429703 (55:2714)

[5] B. C. Berndt, Periodic Bernoulli numbers, summation formulas and applications, Theory and application of special functions, Academic Press, New York, 1975. MR0389729 (52:10560)

[6] B. C. Berndt, Character analogues of the Poisson and Euler-MacLaurin summation formulas with applications. J. Number Theory 7 (1975), 413-445. MR0382187 (52:3075)

[7] B. C. Berndt, Classical theorems on quadratic residues, Enseign. Math. (2) 22 (1976), 261304. MR0441835 (56:229)

[8] B. C. Berndt and M. I. Knopp, Hecke's theory of modular forms and Dirichlet series, World Scientific, Singapore, 2008. MR2387477 (2009a:11003)

[9] S. Bochner, Some properties of modular relations. Ann. of Math. (2) 53 (1951), 332-363. MR0047719 (13:920b)

[10] P. E. Böhmer, Differenzengleichungen und Bestimmte Integrale, Koecher Verlag, Berlin, 1939.

[11] L. Carlitz, Arithmetic properties of generalized Bernoulli numbers, J. Reine Angew. Math. 202 (1959), 174-182. MR0109132 (22:20)

[12] K. Chakraborty, S. Kanemitsu and H. Tsukada, Arithmetical Fourier series and the modular relation, to appear. 
[13] P. Chowla, On the class-number of real quadratic fields, J. Reine Angew. Math. 230 (1968), 51-60. MR0225752 (37:1345)

[14] K. Dilcher, L. Skula and I. Sh. Slavutsukii, Bernoulli Numbers Bibliography (1713-1990). Queen's University Press, Kingston, ON, 1991. MR,1119305 (92f:11001)

[15] H. Hasse, Vorlesungen über Zahlentheorie, 2 auf., Springer, Berlin-New York-Heidelberg, 1964. MR0188128(32:5569)

[16] T. Ishii and T. Oda, A short history on investigation of the special values of zeta and $L$-functions of totally real number fields, in Proc. of the conf. in memory of Tsuneo Aarakawa, Automorphic forms and zeta functions, World Sci., Singapore, 2006. MR 2208776 (2007b:11180)

[17] K. Iwasawa, Lectures on p-adic L-functions, Ann. Math. Studies 74, Princeton Univ. Press, Princeton, 1972. MR0360526 (50:12974)

[18] W. Johnson and K. J. Mitchell, Summation of sums of the Legendre symbols, Pacific J. Math. 69 (1977), 117-124. MR0434936 (55:7899)

[19] H. Joris, On the evaluation of Gaussian sums for non-primitive Dirichlet characters, Enseign. Math. (2) 23 (1977), 13-18. MR0441888 (56:279)

[20] S. Kanemitsu and Kuzumaki, Transformation formulas for Lambert series, Siaulai Math. Sem. 4 (2009), 105-123. MR.2530201

[21] S. Kanemitsu, J. Ma and Y. Tanigawa, Arithmetical identities and zeta-functions, Math. Nachr., to appear.

[22] S. Kanemitsu, J. Ma and W.-P. Zhang, On the discrete mean value of the product of two Dirichlet L-functions, Abh. Math. Sem. Univ. Hamburg 79 (2009), 149-164. MR2545597

[23] S. Kanemitsu, Y. Tanigawa and M. Yoshimoto, Ramanujan's formula and modular forms, in Number-theoretic methods - future trends (ed. by Shigeru Kanemitsu and Chaohua Jia), Kluwer Academic Publ., 2002, pp. 159-212. MR.1974140 (2004g:11036)

[24] S. Kanemitsu and H. Tsukada, Vistas of special functions, World Scientific, SingaporeLondon-New York, 2007. MR2352572

[25] S. Kanemitsu, J. Urbanowicz and N. -L. Wang, Class number formula of a certain imaginary quadratic field, to appear.

[26] M. I. Knopp, Hamburger's theorem on $\zeta(s)$ and the abundance principle for Dirichlet series with functional equations, Number Theory, ed. by R. P. Bambah et al., Hindustan Books Agency, 2000, 201-216. MR.1764804 (2001h:11112)

[27] D. S. Kubert and S. Lang, Modular units, Springer-Verlag, New York-Berlin, 1981. MR648603 (84h:12009)

[28] H. W. Leopoldt, Eine Verallgemeinerung der Bernoullischen Zahlen, Abh. Math. Sem. Univ. Hamburg 22 (1958), 131-140. MR0092812 (19:1161e)

[29] M. Lerch, Essais sur le calcul de nombre de classe de formes quadratiques binaires aux coefficients entiers, Acta Math. 29 (1905), 333-424; 30 (1906), 203-293.

[30] J. Milnor, On polylogarithms, Hurwitz zeta-functions and the Kubert identities, Enseign. Math. (2) 29 (1983), 281-322. MR719313 (86d:11007)

[31] Y. Morita, On the Hurwitz-Lerch L-function, J. Fac. Sci. Univ. Tokyo Sect. IA Math. 24 (1977), 29-43. MR0441924 (56:315)

[32] J. Neukirch, The Beilinson conjecture for algebraic number fields, in "Beilinson's conjectures on special values of $L$-functions", ed. by M. Rapoport et al., Academic Press, Boston, 1988, 193-247. MR944995 (90f:11042)

[33] J.-P. Serre, A course in arithmetic, Springer-Verlag, Berlin-Heidelberg-New York, 1973. MR0344216 (49:8956)

[34] A. Schinzel, J. Urbanowicz and P. van Wamelen, Class numbers and short sums of Kronecker symbols, J. Number Theory 78 (1999), 62-84. MR.1706925 (2000g:11103)

[35] J. Szmidt, J. Urbanowicz and D. Zagier, Congruences among generalized Bernoulli numbers, Acta Arith. 3 (1995), 273-278. MR1339132 (96f:11032)

[36] H. S. Vandiver, An arithmetical theory of the Bernoulli numbers, Trans. Amer. Math. Soc. 51 (1942), 502-531. MR0006742(4:34e)

[37] N. -L. Wang, J. -Z. Li, D. -S. Liu, Euler number congruences and Dirichlet L-functions, J. Number Theory 129 (2009), 1522-1531. MR2521491 (2010c:11032)

[38] Y. Yamamoto, Dirichlet series with periodic coefficients, Proc. Intern. Sympos. "Algebraic Number Theory", Kyoto, 1976, 275-289. JSPS, Tokyo, 1977. MR0450213 (56:8509) 
Graduate School of Advanced Technology, Kinki University Iizuka, Fukuoka, Japan, 820-8555.

E-mail address: kanemitu@fuk.kindai.ac.jp

Department of Mathematics, Weinan Teachers College, Weinan, People's Republic OF CHINA, 714000.

E-mail address: lihailong@wntc.edu.cn

Institute of Mathematics, Shangluo University, Shangluo Shannxi 726000, People's RePUBlic of China

E-mail address: wangnianliangshangluo@yahoo.com.cn 